\title{
Reconfigurable all-diffractive optical filters using phase-only spatial light modulators
}

\author{
Gladys Mínguez-Vega, ${ }^{1,2, *}$ V. R. Supradeepa, ${ }^{3}$ Omel Mendoza-Yero, ${ }^{1,2}$ and Andrew M. Weiner ${ }^{3}$ \\ ${ }^{1}$ Grup De Recerca d'Òptica de Castelló, Departament de Física, Universitat Jaume I, 12080 Castelló, Spain \\ ${ }^{2}$ Institut de Noves Tecnologíes de la Imatge (INIT), Universitat Jaume I, 12080 Castelló, Spain \\ ${ }^{3}$ School of Electrical and Computer Engineering, Purdue University, West Lafayette, Indiana 47907, USA \\ *Corresponding author: gminguez@uji.es
}

Received April 16, 2010; revised June 24, 2010; accepted June 24, 2010;

posted June 29, 2010 (Doc. ID 127146); published July 9, 2010

\begin{abstract}
We demonstrate a reconfigurable optical filter implemented using a phase-only two-dimensional liquid-crystalon-silicon spatial light modulator. To achieve this we utilize two different approaches leading to two different configurations in the modulator. The first one, based on a spatially patterned diffractive lens, permits us to obtain the desired spectrum along the optical axis and, in the second one, which is based on a generalized spectrometer, the desired spectrum is found outside of the optical axis. Experimental results show good agreement with the theory and indicate the validity of this technique. (c) 2010 Optical Society of America

OCIS codes: $\quad 050.0050,120.6200,130.3120$.
\end{abstract}

Optical filters based on diffractive optical elements (DOEs) have received increased attention since the development of the first synthetic spectrum as a tool for correlation spectroscopy [1]. The generation of a synthetic spectrum requires the design of a DOE that transforms the spectrum of the incident light into the spectrum of interest. Based on this idea, several approaches have been reported in the literature. DOEbased spectral synthesis has been performed by means of generalized spectrometers [2], digital mirror arrays [3], micromechanical grating arrays [4], micromechanical diffractive lenses (DLs) [5], moving microgratings [6], or spatially patterned kinoform DLs [7]. In general, these systems are complex, requiring the use of diffractive, refractive, and, in some cases, expensive high-technology components that maybe difficult to align. The spatial light modulator (SLM) could provide an opportunity to change such a situation, because it allows encoding the suitable spatial patterns to generate the desired spectrum in a compact and inexpensive manner. In this Letter, we demonstrate that a single phase-only two-dimensional (2D) SLM can be used as an optical filter. To increase the flexibility, we implemented two different configurations in the SLM. The first one is based on spatially patterned DLs [7], and the second one is based on an all-diffractive generalized spectrometer $[2,8]$.

As a first optical filter, we consider the system based on a spatially patterned DL [7]. This device has also shown its potential as a quasi-direct space-to-time pulse shaper [9]. We will first start with a simple formulation for this filter, where we will assume that the DL and the mask are independent entities. For this setting, we will describe a methodology for obtaining the corresponding complex mask function having both amplitude and phase information. We will then describe the procedure used to integrate this mask and the DL in the SLM as a single phase pattern.

The optical filter based on a spatially patterned DL consists of a circularly symmetric mask and a DL that face each other. The DL can be approximated by a thin lens with a frequency-dependent focal length of value $Z(\nu)=Z_{o} \nu / \nu_{o}$, where $Z_{o}$ is the focal length for the fre- quency $\nu_{0}$. Up to now, this optical filter has been used with fixed masks. Now, with the SLM, we generate userdefined spectra that can be reconfigured with a refresh rate of $60 \mathrm{~Hz}$ or above.

Let us consider a complex transmittance mask $t(r)=|t(r)| \exp [i \phi(r)]$, with $|t(r)|$ as the amplitude and $\phi(r)$ as the phase of the function. For convenience, we express $t(r)$ as a function of a new variable, defined as $s=r^{2} / a^{2}-0.5$, where $r$ refers to the radial coordinate and $a$ is the maximum extension of the mask function. In such a way we define $q(s)=t(r)$. We use a polychromatic plane wave of power spectrum $S(\nu)$ to illuminate the optical filter constituted by the combination of this mask with a DL. Under this condition, the on-axis output irradiance $I_{\text {out }}(\nu)$ at the focal plane of the system, located at a distance $Z_{o}$ from the DL, is given by

$$
I_{\text {out }}(\nu)=\left(\frac{\pi \nu a^{2}}{Z_{o} c}\right)^{2} S(\nu)\left|\tilde{Q}\left(\frac{a^{2}\left(\nu-\nu_{o}\right)}{2 Z_{o} c}\right)\right|^{2},
$$

where $\tilde{Q}(u)=\int_{-\infty}^{\infty} q(s) \exp [i 2 \pi u s] \mathrm{d} s$ is the Fourier transform (FT) of $q(s)$. If $\Delta \nu \ll \nu_{o}$, the leading $\nu$ in Eq. (1) can be approximated by $\nu_{0}$. If this approximation does not hold, the influence of the $\nu$ factor can be precompensated in the mask design.

Now we discuss the mask design. To find a finite aperture complex mask corresponding, through Eq. (1), to a user-defined spectrum, we use the four-step iterative algorithm described in [10]. We illustrate our procedure with an example in which we choose a $\tilde{Q}(u)$ function that corresponds to two equal amplitude Gaussian functions. In view of our system parameters, we choose a target comprising two peaks of the same height centered at 1550 and $1592 \mathrm{~nm}$ with an FWHM of about $11.5 \mathrm{~nm}$. Figures $1(\mathrm{a})$ and $1(\mathrm{~b})$ show the profiles of the mask function $q(s)$ for the amplitude and the phase, respectively. After the conversion from the $s$ to the $r$ coordinate and the construction of the 2D mask, the amplitude and the phase distribution for the mask function $t(r)$ can be shown in Figs. 1(c) and 1(d), respectively.

The experimental setup is basically composed of a laser system, an SLM, and an optical spectrum analyzer. 

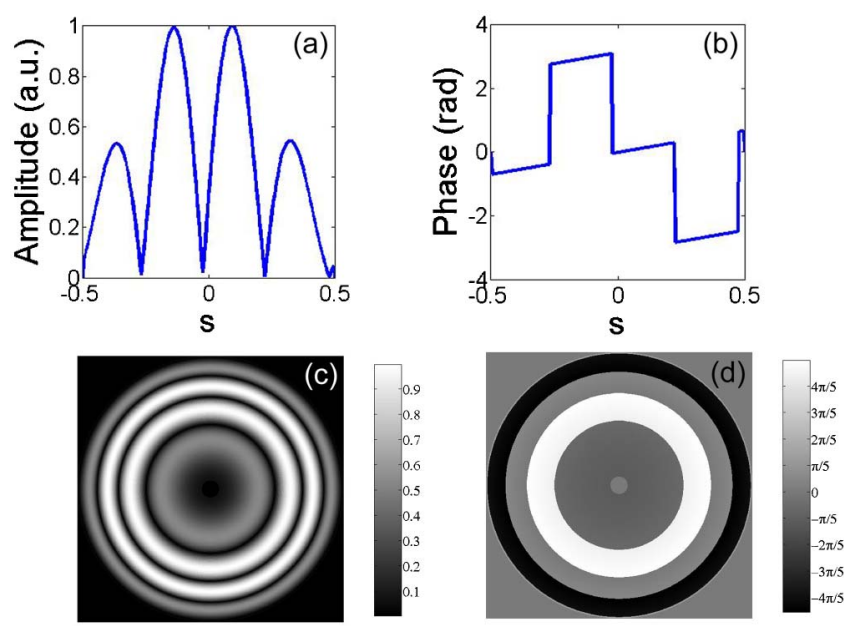

Fig. 1. (Color online) Profile of the mask function in the normalized coordinate $s$ for (a) the amplitude and (b) the phase distribution. 2D circular symmetric mask in the radial coordinate for (c) amplitude and (d) phase distribution.

The laser system is a passively mode-locked erbium fiber laser with a center wavelength of $1570 \mathrm{~nm}$ that generates pulses with a repetition rate of $50 \mathrm{MHz}$. The laser radiation is coupled from fiber to free space by using a standard collimator and is sent through a free-space polarizer. The polarizer allows us to select the correct polarization state required to match the sensitive axis of the SLM. A fiber-based polarization controller is used before the collimator to ensure that maximum power is transmitted through the polarizer. Light then passes through a $10 \times$ beam expander, an iris, and a beam splitter (BS). The transmitted beam from the BS is used to illuminate the SLM. It has a relatively flat intensity profile and a diameter equal to the smaller dimension of the SLM. The SLM, working in reflection mode, allows flexible beam shaping. It is a phase-only liquid-crystal-on-silicon device (HEO $1080 \mathrm{P}$, Holoeye) that provides $>2 \pi$ phase modulation over our bandwidth. It was calibrated for $1570 \mathrm{~nm}$ using a standard polarimetric setup [11]. The beam is then reflected in the BS and sent back into a bare fiber tip of standard single-mode fiber (core size of $\sim 9 \mu \mathrm{m}$ ). Finally, the output power spectrum is monitored through a commercial optical spectrum analyzer (ANDO AQ6317).

To encode a complex-valued function [the complex mask transmittance $t(r)$ together with the DL] onto a phase-only SLM, several procedures have been proposed in the literature. We follow the one reported in [12]. In this technique, a spatially modulated phase is encoded onto the SLM. For each pixel of the SLM, the encoded phase function is obtained by multiplying the desired amplitude and the phase, i.e., the phase transmission function of the SLM is $T(r)=\exp \left[i|t(r)|\left(\phi(r)+\phi_{\mathrm{DL}}\right)\right]$, where $\phi_{\mathrm{DL}}=\exp \left[i \pi \nu_{0} r^{2} / Z_{0} c\right]$ is the phase introduced by the DL. In our case, $Z_{o}=4 \mathrm{~cm}$ for the wavelength of $\lambda_{o}=$ $1570 \mathrm{~nm}$, and we define the mask aperture by setting $|t(r)|=0$ for $r>4.3 \mathrm{~mm}$. The amplitude of the mask transmittance must be defined within the range $[0,1]$, whereas the total phase is defined in the range $[-\pi, \pi]$. As the depth of the phase distribution changes with $|t(r)|$, we spatially modify the diffraction efficiency of the pattern. Mathematically this approach can be demonstrated by using a mixed Fourier-Taylor series to expand $T(r)$ and interpreting this expansion as the formation of different diffraction orders [12]. In our case, each diffraction order will focus in a different plane along the axis. In the first diffraction order, localized at the focal distance of the DL, we place the output fiber to obtain the shaped spectrum. The main limitation of this technique is that a sinc amplitude modulation can appear in the series expansion. Although this distortion can be precompensated [12], in our case it was negligible. Figure 2(a) shows the central pixels of the phase transmission function encoded in the SLM following this procedure for the mask function shown in Fig. 1.

The theoretical and experimental output power spectra are shown in Fig. 3 (top) using continuous and dotted curves, respectively. The dashed curve is the input power spectrum of the laser. In the theoretical spectrum, we incorporated the effect of the power spectrum of the incoming pulse, which causes a reduction of the peak height for the shorter wavelengths. Figure 3(a) corresponds to the output power spectrum obtained for the masks shown in Fig. 2(a). In Fig. 3(b), we adjust the output spectrum to be three Gaussian functions in $\tilde{Q}(u)$, for our system parameters corresponding to equal amplitude peaks centered at 1546, 1570, and $1594 \mathrm{~nm}$, with an FWHM of about $10 \mathrm{~nm}$. The measured spectrum is again close to the target.

As a second configuration, we implement in the SLM a modified version of a generalized spectrometer $[2,8]$. A generalized spectrometer is composed of a diffractive grating (DG) attached to a spatially patterned mask, $m(x)=|m(x)| \exp [i \phi(x)]$, and a refractive lens that focuses the light in a fiber spectrometer. This device has also shown its potential as a direct space-to-time pulse shaper [13]. The key development in our work with respect to previously reported literature is that we emulate the refractive lens by a suitable DL and we implement everything, the DG, the DL, and the mask, all together in the SLM to create a compact system.

A key difference that arises when we use a DL instead of a refractive lens here is that both the DL and the DG are dispersive elements so it is necessary to implement the device in such a way that the dispersive action of the DL can be neglected in comparison with that given by the DG. To this end, we take into account that the maximum spectral resolution of a DG spectrometer is provided by $\Delta \lambda_{\mathrm{DG}} \propto \lambda_{o} p / d$, with $\lambda_{o}$ as the center wavelength, $p$ as the period of the DG, and $d$ as the grating size. In the case of a DL spectrometer, the maximum spectral resolution is provided by $\Delta \lambda_{\mathrm{DL}} \propto \lambda_{o}^{2} 2 Z_{o} / a^{2}$, where $a$ is the maximum
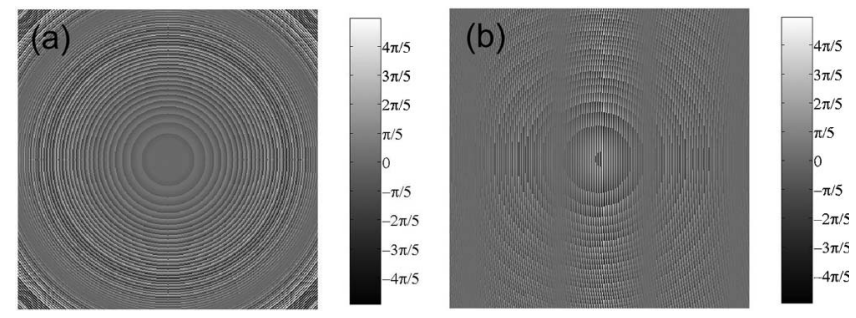

Fig. 2. Phase transmission function encoded in the central $500 \times 500$ pixels of the SLM for (a) the spatially patterned DL and (b) the generalized-spectrometer-based optical filters. 

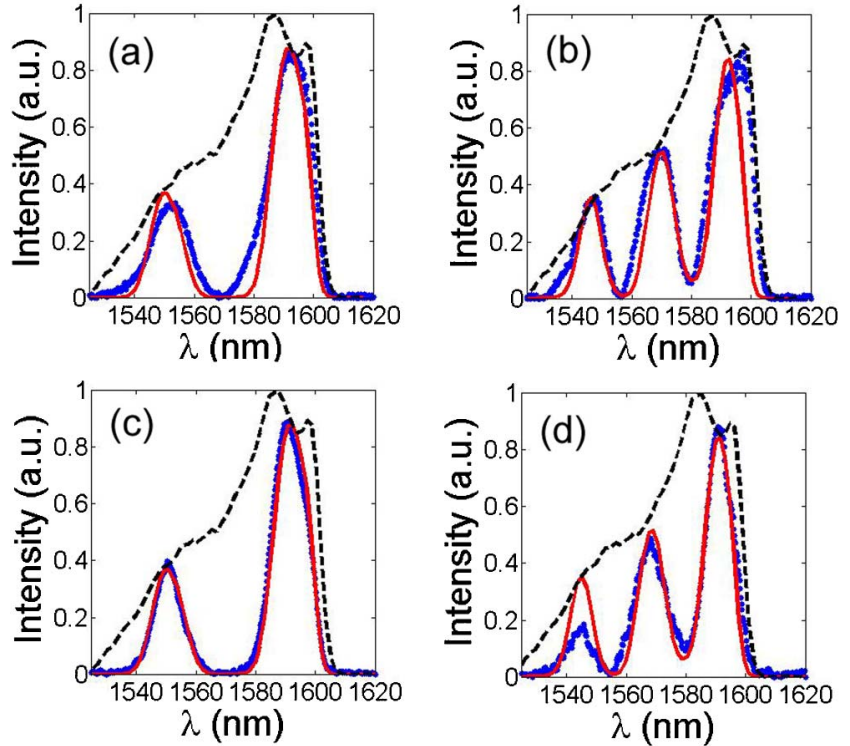

Fig. 3. (Color online) Measured (dotted curve) and theoretical (solid curve) power spectra obtained (a), (b) with the spatially patterned DL optical filter [(a) two-peak spectrum, (b) threepeak spectrum] and (c), (d) with the generalized spectrometer optical filter [(c) two-peak spectrum, (d) three-peak spectrum]. The dashed curve corresponds to the input power spectrum.

radial extension of the DL. This means that we require a large focal distance for the DL and small period for the DG to have $\Delta \lambda_{\mathrm{DL}} \gg \Delta \lambda_{\mathrm{DG}}$. Using normal incidence to the grating, the output power spectrum located at the position of the first diffraction order of the DG for the mean frequency $\nu_{0}$ is then

$$
I_{\text {out }}(\nu) \propto S(\nu)\left|\tilde{M}\left(\frac{\left(\nu-\nu_{o}\right)}{p \nu_{o}}\right)\right|^{2},
$$

when plane-wave illumination is considered. In Eq. (2), $\tilde{M}$ is the FT of the mask function $m(x)$.

To encode in the phase-only SLM the complex-valued function constituted by the one-dimensional (1D) mask, the 2D DL, and the 1D DG, we follow again the procedure employed in [12]. Now, the phase transmission function of the SLM is provided by $T=\exp [i|m(x)|(\phi(x)+$ $\left.\left.\phi_{\mathrm{DL}}+\phi_{\mathrm{DG}}\right)\right]$, where $\phi_{\mathrm{DG}}=\exp [i 2 \pi x / p]$. As an example, Fig. 2(b) shows the central pixels of the phase transmission function encoded in the SLM to obtain the same target as with the mask shown Fig. 2(a). Now we consider experimental parameters of $Z_{o}=25 \mathrm{~cm}$ for the wavelength of $\lambda_{o}=1570 \mathrm{~nm}$ and $p=32 \mu \mathrm{m}$. This guarantees that $\Delta \lambda_{\mathrm{DL}} \gg \Delta \lambda_{\mathrm{DG}}$. Let us remark that, with this configuration, the output point is located in the first diffraction order of the DG, so it is shifted off-axis by the distance $x_{o}=\lambda f / p$, which corresponds here to about $1.2 \mathrm{~cm}$.

For the same targets as above, the desired output power spectra, the corresponding experimental data, and the input power spectrum of the laser are shown in Figs. 3(c) and 3(d) with solid, dotted, and dashed curves, respectively. We show a good agreement between the expected patterns and the measured ones. We believe that the peak height discrepancies in Fig. 3(d) are mainly due to a slight misalignment of the output fiber position.

In conclusion, we have designed and verified experimentally a compact, versatile, and real-time tunable optical filter using a phase-only SLM. We have performed the same experiments with two different configurations in the SLM. The configuration based on the spatially patterned DL allows us to work along the optical axis and is more compact. The setup based on the generalized spectrometer works off-axis and has higher spectral resolution. In both setups, it would be possible to improve performance by using an active feedback that adjusts the gray levels applied on each pixel, in order to match the target filter. This research promises to have impact in different fields whenever a user-defined spectrum is required.

This research was funded in part by the Spanish Ministerio de Educación y Ciencia (MEC), Spain, through Consolider Programme SAUUL CSD2007-00013 and the project FIS2010-15746. G. Mínguez-Vega acknowledges financial support of the Generalitat Valenciana for grant BEST/2009/135.

\section{References}

1. M. B. Sinclair, M. A. Butler, A. J. Ricco, and S. D. Senturia, Appl. Opt. 36, 3342 (1997).

2. J. D. McKinney and A. M. Weiner, Opt. Express 12, 5022 (2004).

3. M. Kanpczyk, A. Krishnan, L. G. de Peralta, A. A. Bernussi, and H. Temkin, IEEE Photonics Technol. Lett. 17, 1743 (2005).

4. H. Sagberg, M. Lacolle, I. R. Johansen, O. Løvhaugen, R. Belikov, O. Solgaard, and A. S. Sudbø, IEEE J. Sel. Top. Quantum Electron. 10, 604 (2004).

5. M. Lacolle, H. Sagberg, I. R. Johansen, O. Løvhaugen, O. Solgaard, and A. S. Sudbø, IEEE Photonics Technol. Lett. 17, 2622 (2005).

6. G. Zhou and F. S. Chau, Opt. Express 16, 9132 (2008).

7. G. Mínguez-Vega, O. Mendoza-Yero, E. Tajahuerce, J. Lancis, and P. Andrés, IEEE Photonics Technol. Lett. 21, 347 (2009).

8. D. Leaird and A. M. Weiner, IEEE J. Quantum Electron. 37, 494 (2001).

9. G. Mínguez-Vega, O. Mendoza-Yero, J. Lancis, R. Gisbert, and P. Andrés, Opt. Express 16, 16993 (2008).

10. J. A. Davis, C. S. Tuvey, O. López-Coronado, J. Campos, M. J. Yzuel, and C. Iemmi, Opt. Lett. 32, 844 (2007).

11. A. M. Weiner, Ultrafast Optics (Wiley, 2009).

12. J. A. Davis, D. M. Cottrell, J. Campos, M. J. Yzuel, and I. Moreno, Appl. Opt. 38, 5004 (1999).

13. D. E. Leaird and A. M. Weiner, Opt. Lett. 24, 853 (1999). 